\title{
PHOTOMETRIC DETERMINATION OF THIOGLYCOLIC ACID IN COSMETICS BY USING A STOPPED-FLOW REVERSE FLOW-INJECTION SYSTEM AND THE FORMATION OF GOLD NANOPARTICLES
}

\author{
Marina Sierra-Rodero, Juan Manuel Fernández-Romero, Agustina Gómez-Hens \\ Department of Analytical Chemistry, Marie Curie Annex Building, Campus of Rabanales, University of Córdoba, E- \\ 14071-Córdoba, Spain. Phone: 34-957218645, Fax: 34-957218644 \\ e-mail: q22sirom@uco.es, web site: http://www.uco.es/investiga/grupos/FQM-303
}

The positive effect of thiol compounds as reducing agents in the synthesis of gold nanoparticles (AuNPs) in the presence of a micellar medium of Triton X-100 (TX-100) has been photometrically studied using a reverse flow-injection system which operates in the stopped-flow mode (SF-rFIA) (Figure A). The reaction of synthesis consists on the injection of tetrachloroauric acid $\left(\mathrm{HAuCl}_{4}\right)$ in a mixture of the thiol and the surfactant. The reaction plug is stopped in a thermostatic bath at $90{ }^{\circ} \mathrm{C}$ during 4 minutes and then is translated to the photometer where the signal is measured at $\lambda=540 \mathrm{~nm}$. The analytical usefulness of this new system has been assessed by its application to the determination of thioglycolic acid (TGA), which was chosen as analyte model.
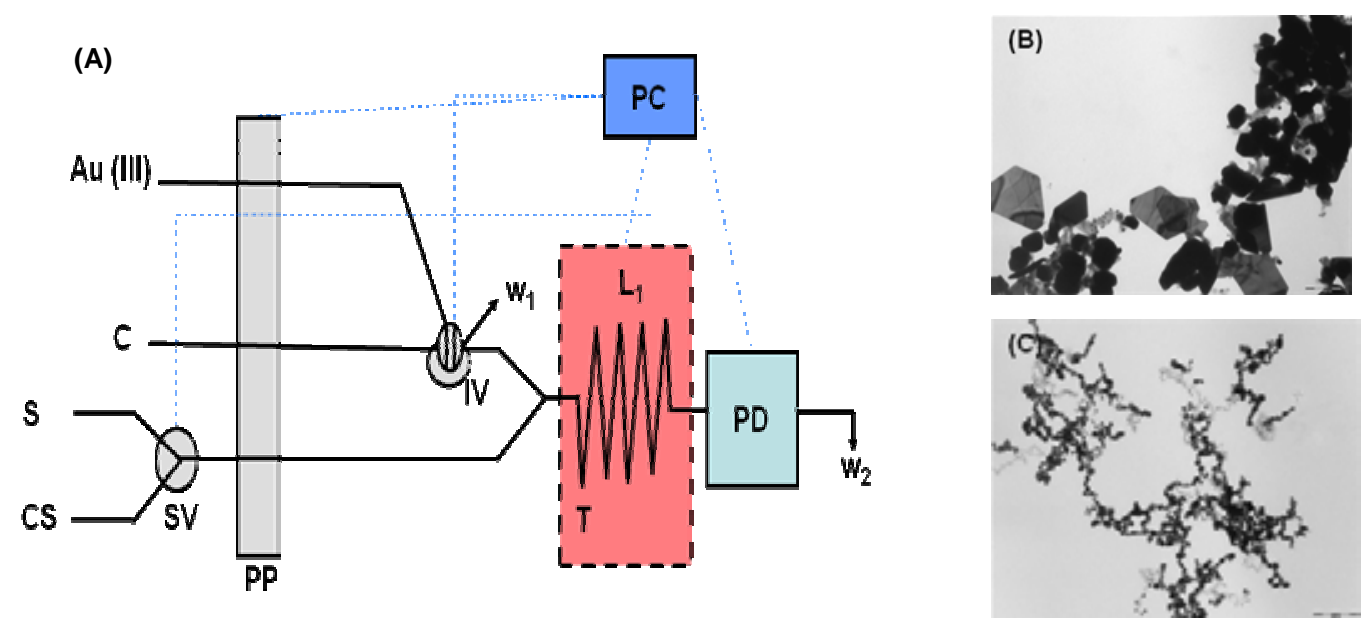

Figures $\mathrm{B}$ and $\mathrm{C}$ depict the TEM images obtained for the mixture of $\mathrm{HAuCl}_{4}$ and TGA in the absence and in the presence of TX-100 respectively. Figure B shows the formation of irregular polyhedral metallic clusters with a wide range of sizes, estimated between 60 and $300 \mathrm{~nm}$. However the TEM image obtained in the presence of TGA and TX-100 shows the formation of spherical AuNPs with sizes in the range 10$40 \mathrm{~nm}$.

The formation of AuNPs increases in a linear mode with the concentration of TGA. The dynamic range of the calibration graph was $5.97-80 \mu \mathrm{mol} \mathrm{L}{ }^{-1}$, and the detection limit was $1.73 \mu \mathrm{mol} \mathrm{L} \mathrm{L}^{-1}$. Other thiol compounds (cysteine, N-acetylcysteine and homocysteine) and related compounds (methionine and homocystine) gave similar results, but gluthatione did not give any signal. The precision of the method, expressed as relative standard deviation, ranged between 1.5 and $2.3 \%$. The method was applied to the determination of TGA in several cosmetic samples with acceptable recoveries in all instances, which ranged between 90.32 and $101.46 \%$. 